\section{E-Democracy: Erwartungen der Bürger und erste Realisie- rungen. Ein Werkstattbericht.}

\author{
von Gerhard Fuchs und Hans Kastenholz, \\ Akademie für Technikfolgenabschätzung in \\ Baden-Württemberg
}

\begin{abstract}
Manipulation und Wahlbetrug, Verflachung der politischen Debatten assoziieren die einen mit der Idee einer "E-Democracy“. Andere sehen in der ,elektronischen Demokratie" eine Chance für den Dialog zwischen Regierenden und Bürgern. In verschiedenen Projekten versuchte die TA-Akademie zu analysieren, wie die Bürger selbst das demokratische Potenzial des Internets einschätzen und durch welche Charakteristika Partizipation an ersten E-Democracy-Versuchen gekennzeichnet ist.
\end{abstract}

\section{Einleitung}

Es scheint, als ob sich unsere Einschätzung der Folgen, die sich aus der Einführung und Verbreitung neuer Technologien ergeben, immer noch allzu stark am technisch Machbaren orientiert. Die Neigung ist verbreitet, das technisch Mögliche auch in komplexe soziale, politische oder ökonomische Zusammenhänge zu projizieren und als bestimmend für dortige Entwicklungen zu erachten. Determinierende Wirkung wird dann den technischen Neuerungen selbst und nicht den handelnden Personen zugeschrieben, die in jeweils spezifische Kontexte eingebunden sind. Denkmuster dieser Art gehen häufig mit der Erwartung einher, (schon) in (naher) $\mathrm{Zu}-$ kunft sei aufgrund technologischer Veränderungen mit massiven Umwälzungen im jeweils angesprochenen Bereich zu rechnen.

Solche Wahrnehmungs- und Bewertungsmuster sind auch in der Diskussion über die zunehmende elektronische Vernetzung (Internet) und deren mögliche Konsequenzen für Politik und politische Partizipation beobachtbar. Neben Skepsis und Befürchtungen verbinden viele mit der Verbreitung des Internets Hoffnungen auf völlig neue Formen von Politik und Demokratie. So wird argumentiert, dass die nunmehr dem Einzelnen gegebenen Möglichkeiten, mit einer großen Zahl anderer Personen auf Distanz kommunizieren und selbst als „Sender” von Information fungieren $\mathrm{zu}$ können, dazu führen werde, dass in der Politik netzwerkartige Strukturen künftig hierarchische ablösten, dass durch den direkteren Zugang zu Informationen und durch die Erweiterung individueller Beteiligungsmöglichkeiten direktdemokratische Politikformen repräsentative ersetzen und damit auch die Bedeutung von intermediären Institutionen wie z. B. den Parteien geringer würden.

Dieser „optimistischen“, technikzentrierten Sichtweise stehen die Einschätzungen der alten Garde der Partizipationsforscher gegenüber (z. B. Gabriel und Mößner 2002), die davon ausgehen, dass die Bereitschaft zur politischen Partizipation stark von sozioökonomischen Faktoren beeinflusst wird, die sich auch unter dem Einfluss des Internets allenfalls langfristig verändern werden. Das Internet wird überproportional von denjenigen genutzt, die eine bessere Bildung und einen allgemein höheren Status haben (vgl. Schenk und Wolf 2001). Wissenschaftler in dieser Tradition sehen daher keine Substanz hinter der Erwartung, dass sich die Bereitschaft zu und die Strukturen der politischen Partizipation als Resultat des Einflusses des Internets gravierend verändern werden.

In dieser Debatte finden jedoch einige Dinge zu wenig Beachtung. Unklar ist, wie die Bürger selbst als direkt Betroffene über politische Beteiligung mit Hilfe des Internets denken. Welche Möglichkeiten und Grenzen sehen sie? Welche Bedenken und Befürchtungen werden von ihnen geäußert? Wo sehen sie Anwendungsfelder elektronischer Demokratie? Gibt es Regulierungswünsche? Wird das Medium Internet zum Zweck der politischen Beteiligung überhaupt akzeptiert?

Zum anderen orientieren sich sowohl die Partizipationsforscher wie auch die Propheten der elektronischen Demokratie stark an den traditionellen Formen der politischen Partizipation wie beispielsweise Teilnahme an Wahlen. Sie vernachlässigen Veränderungen, die im politischen Prozess durch die zunehmende Nutzung des Internets entstehen können. So ist man sich mittlerweile darüber einig geworden, dass Online-Wahlen nicht zuletzt auf Grund von Sicherheitsüberlegungen allenfalls mittelfristig durchgeführt werden können. Trotzdem hat das Internet bereits jetzt das Organisationsverhalten von politischen Akteuren verändert. Zudem ist 
die Frage noch offen, inwieweit sich das Internet tatsächlich „veralltäglichen“ wird. Wenn das Internet zu einem in den Alltag integrierten Medium werden sollte wie das Telefon und nahezu alle Schichten der Bevölkerung erreicht, dann stellt sich auch die Frage nach den Auswirkungen auf das Partizipationsverhalten neu.

Die TA-Akademie setzt sich in ihren Projekten intensiv mit diesen offenen Fragen auseinander. Mit Hilfe des Instruments der Bürgerforen soll herausgearbeitet werden, welche Erwartungen die Bürger momentan im Hinblick auf Bürgerbeteiligung über das Internet haben (Abschnitt 2). Durch die Untersuchung innovativer Anwendungsformen wie dem Virtuellen Parteitag von Bündnis 90/Die Grünen in BadenWürttemberg sollen Chancen und Risiken von über das Internet abgewickelten Politikprozessen vertieft herausgearbeitet werden (Abschnitt 3). Eine Untersuchung der verschiedenen Anwendungsformen von electronic voting soll illustrieren, was tatsächlich an neuen Handlungsmöglichkeiten vorhanden ist (Abschnitt 4).

\section{Bürgerbeteiligung im Internet? - Die Position der Bürger}

Im Rahmen eines Forschungsprojektes für das Deutsche Ministerium für Bildung und Forschung (BMBF) hat die TA-Akademie mittels Bürgerforen die Akzeptanz und die Präferenzen von technischen Anwendungen zur elektronischen Demokratie in der Öffentlichkeit erhoben. ${ }^{1}$ Bürgerforen beruhen konzeptionell auf dem Modell der „Planungszelle” von Peter C. Dienel. Hauptziel dieses Verfahrens ist die Einbindung der allgemeinen Öffentlichkeit in Entscheidungsprozesse (Dienel 1997). Die grundlegende Philosophie von Planungszellen ist von dem Wunsch nach fairer Repräsentation aller Betroffenen bei der Entscheidungsvorbereitung und Entscheidungsfindung getragen.

Eine Planungszelle ist eine Gruppe von 25 Bürgerinnen und Bürgern, die mit Hilfe eines Zufallsverfahrens ausgewählt worden sind. Die BürgerInnen werden für eine begrenzte Zeit (in der Regel 2 bis 4 Tage) von ihren alltäglichen Verpflichtungen freigestellt, um Lösungsmöglichkeiten für vorgegebene Planungs- bzw. Entscheidungsprobleme $\mathrm{zu}$ erarbeiten. Für diese Tätigkeit wird eine angemessene Aufwandsentschädigung gezahlt. Die Arbeit in der Planungs- zelle wird im Vorfeld detailliert vorstrukturiert und von Prozessbegleitern/-begleiterinnen betreut und unterstützt. Die erforderlichen Sachinformationen werden von Experten vermittelt. Bei Interessenkonflikten haben die Vertreter der unterschiedlichen Interessen Gelegenheit, ihre spezifische Sichtweise zu formulieren. Die im kontinuierlichen Diskurs der Planungszelle erarbeiteten Problemlösungs- bzw. Handlungsvorschläge und Entscheidungsanregungen werden als Bürgergutachten zusammengefasst und veröffentlicht (Bongardt 1999).

Vor diesem Verfahrenshintergrund wurden von der TA-Akademie im Mai 2001 fünf Bürgerforen zum Thema „Bürgerbeteiligung im Internet? Möglichkeiten und Grenzen elektronischer Demokratie" durchgeführt (Kastenholz und Wienhöfer 2002). Durchführungsorte waren die baden-württembergischen Städte $\mathrm{Bad}$ Schussenried, Ettenheim, Mannheim, Stuttgart und Weikersheim. Die Auswahl der Städte erfolgte nach Anzahl der Einwohner und nach geographischer Lage (städtischer bzw. ländlicher Raum). Die Teilnehmer an den Bürgerforen wurden nach dem Zufallsprinzip ausgewählt. Hierzu wurden die Einwohnermeldeämter der Durchführungsorte angeschrieben und um 600 zufällig ausgewählte Anschriften von Einwohnern gebeten, die älter als 18 Jahre sein sollten. Die so ausgewählten Bürgerinnen und Bürger wurden schriftlich zur Teilnahme eingeladen.

Letztlich wurden vom Projektteam folgende von den Teilnehmern der Bürgerforen konkret zu bearbeitende Fragestellungen und Gruppenaufgaben entwickelt:

- Bestandsaufnahme von wahrgenommenen signifikanten Demokratie- und Kommunikationsdefiziten des gegenwärtigen politischen Systems,

- Möglichkeiten und Grenzen einer politischen Partizipation von Bürgerinnen und Bürgern durch das Internet,

- Möglichkeiten und Grenzen der Verbesserung der Kommunikation zwischen Bürger und Politik durch das Internet,

- vorstellbare Anwendungsmöglichkeiten elektronischer Demokratie,

- gewünschte Themenschwerpunkte für elektronische Demokratie,

- mögliche Erfolgsaussichten von zeitlich befristeten Bürgernetzwerken im Internet, 
- Probleme der Chancengleichheit bei elektronischer Demokratie,

- Probleme der Datensicherheit bei Abstimmungen und Wahlen mit dem Internet,

- politische, institutionelle und individuelle Voraussetzungen für die Anwendung von Formen elektronischer Demokratie.

Zur Bearbeitung der Aufgaben hatten die Teilnehmer zwei Tage Zeit. Zu Beginn erhielten sie von einer Expertin eine Einführung in die aktuelle Diskussion um „EDemokratie“, sowie eine Darstellung ihrer historischen Wurzeln. Möglichkeiten der politischen Beteiligung per Internet wurden vorgestellt und anhand praktischer Beispiele diskutiert. Ebenso erhielten die Teilnehmer eine kurze Einführung in die Funktionsweise und in die Eigenschaften des Internets.

Zusätzlich wurden vom Projektteam verschiedene E-Demokratie-Projekte aus den Bereichen Stadtplanung, Verwaltung (Elektronische Dienste) und Parteiprogrammgestaltung am Computer vorgestellt und erläutert, so dass sich die Teilnehmer direkt ein Bild davon machen konnten, welche Möglichkeiten der Interaktion und der Partizipation am Internet bestehen. Außerdem hatten die Teilnehmer auch selbst die Gelegenheit, sich an den Computer $\mathrm{zu}$ setzen und per Mausklick das Internet und die Referenzprojekte ,auszuprobieren“.

Die Ergebnisse der einzelnen Bürgerforen wurden von einem Redaktionsteam ausgewertet und in Form eines vorläufigen Empfehlungskatalogs aufgearbeitet. Dieser wurde im Juli 2001 gemeinsam mit den Delegierten der einzelnen Foren nochmals hinsichtlich Korrektheit und Vollständigkeit diskutiert und abschließend gemeinsam verabschiedet. Die wichtigsten Ergebnisse können wie folgt zusammengefasst werden (Wienhöfer, Kastenholz und Geyer 2002):

Mit Ausnahme von Themen, die in der Regel in der Öffentlichkeit vorwiegend populistisch abhandelt werden und die Emotionen gegen Minderheiten wecken oder schüren (z. B. Diskussionen über Todesstrafe, Asylrecht, Ausländerzuzug) werden alle politischen Themen auf allen institutionellen Ebenen (EU, Bund, Länder, Kommunen) als geeignet für Diskussionen in dem Medium Internet erachtet. Jedoch wird die kommunale Ebene bevorzugt, weil hier bürgerschaftlicher Sachverstand am ehesten wirksam werden kann und weil hier zumindest in der Anfangsphase elektronischer Demokratie Grenzen und Risiken überschaubar bleiben.

Chancen erkennen die Bürgerforen im Potenzial des Internets, den politischen Kommunikations- und Informationsfluss effizienter und transparenter zu gestalten sowie die Partizipationsmöglichkeiten der Bürger an politischen Prozessen $\mathrm{zu}$ verbessern. Im Vordergrund stehen hierbei:

- effizientere Administration durch virtuelle Behördengänge,

- effizientere Kommunikation zwischen Politik und Bürgerschaft,

- Aktivierung und Motivierung durch das Internet auch für partizipatives politisches Engagement außerhalb des Mediums Internet,

- lebensnähere politische Entscheidungen durch Einbezug bürgerschaftlichen Erfahrungswissens.

Risiken erkennen die Bürgerforen im Blick auf elektronische Demokratie vor allem in ungelösten Problemen wie:

- Gefahr der Manipulation bei Abstimmungen und Wahlen aufgrund noch nicht ausreichender Datensicherheit,

- Gefahr der gesellschaftlichen Spaltung in Informierte und Uninformierte (digitale Kluft) und damit Beeinträchtigung der demokratischen Chancengleichheit,

- Gefahr der Trivialisierung der Politik durch mögliche Beeinflussung politischer Entscheidungen durch $\mathrm{zu}$ viele spontane und unreflektierte Meinungsbilder,

- Gefahr der Propaganda krimineller und extremistischer Gruppen und ihrer Einflussnahme insbesondere auf junge Menschen.

Als konkrete Anwendungsmöglichkeiten von Formen elektronischer Demokratie wünschen sich die Bürgerforen in erster Linie:

- Zurverfügungstellung elektronischer Dienste durch die Verwaltung (Steuer, Passausstellung etc.),

- Bürgernah aufbereitete, zeitnahe und umfassende Informationen z. B. über Flächennutzungspläne, Gemeinderatsprotokolle etc. auf kommunaler Ebene sowie z. B. über Gesetzesinitiativen, Abstimmungsergebnisse auf allen politischen Ebenen, 
- Etablierung von Bürgernetzwerken (Bürgerinitiativen, Unterschriftensammlungen, Elterninitiativen, Nachbarschaftshilfe),

- Möglichkeit der Online-Kommunikation (Fragestunden, Diskussionsforen etc.).

Verbindliche Abstimmungen über das Internet im Sinne einer direkten Demokratie wurden in allen fünf Bürgerforen kontrovers diskutiert. Hingegen wurden Plebiszite zu ethisch umstrittenen Themen (z. B. Todesstrafe) und zu komplexen Entscheidungen, die eine vertiefte Einarbeitung in die Materie erfordern, einmütig abgelehnt. In Verbindung mit einer verlässlichen Identifizierung mittels einer digitalen Signatur halten es die Bürgerforen jedoch ebenfalls einmütig für sinnvoll, zu aktuellen politischen Diskussionen Meinungsbilder durch OnlineAbstimmungen einzuholen.

Wählen vom häuslichen Computer aus ist in den Bürgerforen ebenfalls umstritten. Manche Bürger sehen darin die Chance zur Beseitigung der Wahlmüdigkeit (insbesondere bei jungen Menschen). Andere lehnen es jedoch aus Überlegungen zur politischen Kultur und zur Authentizität der abgegebenen Stimmen ab. Konsens herrscht jedoch in der Feststellung, dass der derzeitige Sicherheitsstand rechtsgültige Online-Wahlen ohnehin noch nicht zulässt.

Als Grundvoraussetzungen und Rahmenbedingungen für die Einführung und die Nutzung von Formen elektronischer Demokratie wurden von den Bürgern folgende Punkte als besonders wichtig erachtet:

- Die Bereitschaft der Bürger zum politischen Engagement und zur Partizipation setzt den Willen der Politiker voraus, größere Transparenz bei politischen Entscheidungen zu schaffen und die Bürger hierin mit einzubeziehen.

- Eine breite Akzeptanz elektronischer Demokratie bei den Bürgern kann nur erreicht werden, wenn die Menschen dem neuen Medium ausreichend Vertrauen entgegenbringen. Die Anstrengungen zur Weiterentwicklung sicherheitstechnischer Maßnahmen müssen daher weiter vorangebracht werden, um die Kommunikation und die Datenübermittlung vor Missbrauch und Manipulation zu schützen.

- Bürgerbeteiligung über das Internet ist langfristig nur dann sinnvoll, wenn sie sich nicht auf einzelne Prestigeprojekte konzentriert, sondern umfassend und im Zusammenhang aller politischer Themen eingesetzt wird. Um dies zu gewährleisten, muss die Politik entsprechende strukturelle und juristische Voraussetzungen schaffen.

- Die Gefahr einer digitalen Spaltung der Gesellschaft ist relevant und darf nicht aus den Augen verloren werden. Um ihr zu begegnen, ist eine Förderung der Medienkompetenz erforderlich. Hier sind vor allem die Bildungseinrichtungen gefordert. Ebenso sind die strukturellen Voraussetzungen zu optimieren, die einer breiteren Bevölkerung den Zugang zum Internet ermöglichen.

- Bei allen Überlegungen zur Nutzung von Formen elektronischer Demokratie ist stets daran zu denken, dass alle bisherigen Möglichkeiten der Informationsbeschaffung, der Kommunikation und der Partizipation auch weiterhin in gleichem Umfang außerhalb des Internets gewährleistet bleiben müssen. Das Internet soll immer nur Ergänzung, nie Ersatz sein.

Im Ergebnis kann die Einstellung der Bürgerinnen und Bürger als skeptische Aufgeschlossenheit bezeichnet werden, wobei die Skepsis in dem Maße abzunehmen scheint, wie es den Verantwortlichen gelingt, die als besonders gravierend empfundenen Probleme der Datensicherheit, der digitalen Kluft und der Bereitschaft auf der politischen Seite zur Zulassung von Transparenz und Bürgerpartizipation zu lösen.

\section{Virtueller Parteitag von Bündnis 90/Die Grünen}

Der Eindruck einer grundsätzlichen Aufgeschlossenheit, weit entfernt von Technikskepsis oder Technikablehnung, bestätigt sich auch bei der Evaluierung des Parteitages von Bündnis90/Die Grünen. Mit dem vom 24.11. bis 03.12.2000 veranstalteten Virtuellen Parteitag (ViP) wurde zum ersten Mal in Deutschland ein Parteitag vollständig im Internet abgehalten. Ein wichtiges Ergebnis dieses Experiments ist, dass virtuelle (Politik-)Veranstaltungen schon jetzt interessante Ergänzungen zu herkömmlichen Veranstaltungen darstellen können. Sie sind jedoch nicht geeignet, diese völlig zu ersetzen. Vergleicht man beide Veranstaltungsformen, weisen sie Vorzüge in einigen Bereichen sowie Beschränkungen 
und Nachteile in anderen aus. Insofern wird durch die Evaluation die Einschätzung der BürgerInnen aus den Foren bestätigt, die ebenfalls Chancen als auch Risiken sahen. Was passierte genau auf dem virtuellen Parteitag? ${ }^{2}$

Zunächst ist festzustellen, dass es den Organisatoren gelang, für einen so genannten „kleinen” Landesparteitag mit über 300 Teilnehmern eine hohe Zahl an Mitgliedern zu mobilisieren. Angemeldet, und damit auch zur aktiven Beteiligung berechtigt, waren insgesamt 303 Mitglieder, von denen allerdings ein nicht geringer Teil wegen anderer (beruflicher oder familiärer) Verpflichtungen nicht teilnehmen konnte. Im Gegenzug hat eine größere Zahl von Mitgliedern das Veranstaltungsgeschehen auch ohne Anmeldung (als "Zuschauer") verfolgt. ${ }^{3}$ Bei künftigen virtuellen Parteitagsveranstaltungen könnte diese hohe Teilnehmerzahl aufgrund des geringeren Neuheitswerts abnehmen, sie könnte aber auch durch Weiterentwicklung der Veranstaltungsform und durch verbessertes „Marketing” noch erhöht werden.

Das Experiment „Durchführung eines Parteitags via Internet" hat nahezu ausschließlich Mitgliederschichten angesprochen, die auch vorher schon politisch engagiert gewesen sind. Dies mag auch damit zusammenhängen, dass es nicht das Ziel der Veranstalter gewesen war, vordringlich politisch inaktive Mitglieder zu erreichen. Unsere Untersuchungsergebnisse deuten jedoch darauf hin, dass virtuelle Veranstaltungsorganisation ein Mittel sein kann, (an politischer Aktivität) interessierte Mitglieder zum Besuch von (Partei-) Veranstaltungen zu motivieren. Dafür spricht unter anderem, dass knapp ein Viertel aller Besucher mit dem ViP zum ersten Mal überhaupt einen Parteitag besucht haben (vgl. Bubeck und Fuchs 2001).

Die Altersverteilung des ViP entsprach weitgehend der herkömmlicher Parteitagsveranstaltungen mit einer starken Präsenz der Altersgruppe der 30- bis 49-Jährigen. Frauen waren gegenüber Männern etwas unterrepräsentiert. Der wesentliche Unterschied gegenüber der Besucherschaft herkömmlicher (Parteitags-)Veranstaltungen bestand in der sehr markanten Internet-Affinität der ViP-Besucher. Für die These, dass die immer größere Ausbreitung des Internets in nahezu allen gesellschaftlichen Bereichen die ohnehin schon bestehenden Ungleichheiten in der Gesellschaft noch verstärken könnte, spricht der Tatbestand, dass die ViP-Besucher zu einem sehr hohen Anteil höhere Bildungsabschlüsse haben. Mitglieder mit Hauptschul- als höchstem Schulabschluss oder einfachen beruflichen Tätigkeiten waren unter den ViP-Besuchern wenig oder überhaupt nicht vertreten.

Der größte Vorteil aus der Sicht der Teilnehmer war die Beteiligungsfreundlichkeit des ViP. Dazu zählt, dass die virtuelle Veranstaltung dem ,einfachen” Teilnehmer mehr Möglichkeiten für ein eigenes Aktiv-Werden bot. Auch waren, anders als bei herkömmlichen Parteitagen, keine Beschränkungen vorgegeben, was Länge oder Zahl der Diskussionsbeiträge anbelangte. Besonders wichtig war für viele Teilnehmerinnen und Teilnehmer, dass die Hemmschwelle, sich vor einem großen Publikum zu Wort zu melden, niedriger war. Diese Eigenschaften des ViP führten dazu, dass insgesamt eine große Anzahl an Diskussionsbeiträgen eingebracht wurde und sich ein größerer Teil der Teilnehmer aktiv beteiligte. Beteiligungsfreundlich war der ViP schließlich auch deshalb, weil die Besucher dem ViP in ihrer weit überwiegenden Mehrheit eine bessere Vereinbarkeit mit Familie und Beruf bescheinigten.

Ein wichtiger Bestandteil der Veranstaltung war die Parteitagsdebatte. Neben der niedrigeren Schwelle, sich vor einem großen Publikum zu Wort zu melden, war aus der Sicht der Teilnehmer eine weitere positive Eigenschaft der virtuellen Debatte die größere Ausführlichkeit, mit der Themen verhandelt werden konnten. Von Vorteil empfanden viele auch den geringeren Zeitdruck, unter dem die Diskussion stand dass etwa eigene Äußerungen oder die anderer in Ruhe reflektiert werden konnten. Nach Ansicht von Teilnehmern war der Argumentationsstil der virtuellen Debatte weniger rhetorisch und emotional geprägt als der herkömmlicher Parteitagsdebatten.

Persönliche Kontakte und informeller Austausch kamen aus der Sicht der Teilnehmer im Verlauf des Virtuellen Parteitags deutlich zu kurz. So machte es sich negativ bemerkbar, dass die bereit gestellten Kommunikationsmöglichkeiten vertrauliche Kommunikation zwischen den Teilnehmern nicht erlaubten. Ihnen wurde vor dem Bildschirm auch nicht angezeigt, wer sich zur gleichen Zeit auf der Veranstaltung befand, was das spontane Ansprechen anderer 
Teilnehmer verhinderte. Viele der Befragten empfanden nicht zuletzt aus diesen Gründen während ihres Veranstaltungsbesuchs ein Gefühl der Einsamkeit und des Abgeschnittenseins von den anderen ViP-Besuchern.

Obwohl die Teilnehmer den ViP-Besuch überwiegend als spannendes und anregendes Erlebnis empfanden, vermisste die Mehrheit der Befragten das „sinnliche Erleben”, das mit der physischen Anwesenheit einer größeren Zahl von Menschen verbunden ist. Die „Stimmung” im Tagungssaal, wahrnehmbare Emotionalität, Applaus und Zwischenrufe, die Eindrücklichkeit einer engagierten Rede konnten nach Ansicht Vieler beim ViP nicht ausreichend ersetzt werden. Auch die Wahrnehmung der anderen als „ganzer Person”, die sonst übliche Orientierung an äußeren Merkmalen der Sprechenden, an deren Stimme, Mimik, Gestik und auch deren räumliche Positionierung entfiel. Auf der einen Seite war es für viele ein Vorteil, dass die Bewertung ihrer "Rede“beiträge nicht davon abhing, wie diese ,rübergebracht” wurden, auf der anderen Seite bedeutete der gleiche Umstand aber einen Verlust an Reichhaltigkeit der Sinneseindrücke und der Information.

Die Gesamtverweildauer und die Verteilung der Besuchszeiten des ViP, die innerhalb der Gruppe der Teilnehmer stark variierten, unterschieden sich erwartungsgemäß von „realen” Veranstaltungen. Neben dem Wegfall fester Besuchszeiten bestand ein Hauptunterschied in den überwiegend kürzeren Besuchsepisoden seitens der Teilnehmer. Von großer Bedeutung für das Besuchsverhalten war der Umstand, dass die Teilnahme räumlich nicht getrennt vom Alltag erfolgte und über den Zeitraum von zehn Tagen in den Alltag integriert und gegenüber dessen Ansprüchen ,verteidigt” werden musste. Aufgrund dieses Arrangements mussten die Besucher immer wieder erneut über Zeitpunkt und Häufigkeit ihres Besuchs entscheiden. Auch Meinungsbildung und Rezeption von Diskussionsbeiträgen schien ohne die Möglichkeit der Orientierung am Verhalten der anderen Besucher, aber auch ohne ,soziale Kontrolle" individueller zu erfolgen.

Als der wesentliche Faktor zur Erklärung von Unterschieden in der Wahrnehmung und Bewertung des ViP erwies sich das Alter und nicht, wie man hätte vermuten können, die Internet-Erfahrenheit der Parteitagsbesucher.
Besonders groß waren die Unterschiede in der Wahrnehmung und Bewertung des ViP zwischen den Altersgruppen der unter 30-Jährigen einerseits und derjenigen der 40-Jährigen und Älteren andererseits. Jüngere Teilnehmer beteiligten sich stärker und hatten von verschiedenen Aspekten der virtuellen Veranstaltung durchweg einen positiveren Eindruck als ältere Besucher. 91,7\% der unter 30-Jährigen erklärten sich mit dem ViP insgesamt ziemlich oder sehr zufrieden, bei den 40-Jährigen und Älteren lag dieser Anteil bei ,nur” 55,6\%.

Aus der wissenschaftlichen Begleitung des ViP lassen sich für künftige virtuelle (Partei-) Veranstaltungen eine Reihe von Lehren ziehen. Was die technisch-organisatorische Umsetzung anbelangt, haben Bedienbarkeit und Übersichtlichkeit der Parteitagsseiten, umfassende Information sowie Hilfe- und Betreuungsangebote bei technischen Problemen aus der Sicht der Teilnehmer einen besonders hohen Stellenwert und sollten bei künftigen Veranstaltungen (noch) verbessert bzw. ausgebaut werden. In Hinblick auf die virtuelle Veranstaltung als Ort sozialen Austauschs wären insbesondere die Kommunikationsmöglichkeiten zwischen den Parteitagsbesuchern zu erweitern und $\mathrm{zu}$ verbessern. Besonders positiv aufgenommen wurden von den beteiligten Mitgliedern lokal organisierte „reale” Begleitveranstaltungen etwa im Büro des Ortsvereins oder in einem Internet-Café. Solche Parallel-Angebote haben zum einen den Vorteil, dass sie dem Bedürfnis der Teilnehmer nach Geselligkeit entgegenkommen, sie können aber zugleich auch die Beteiligungsschwellen für die Mitglieder mindern helfen, die noch geringe oder überhaupt keine Internet-Kenntnisse haben.

Die ganz überwiegende Mehrheit der ViPBesucher $(89,7 \%)$ wünscht sich, dass weitere virtuelle Parteitagsveranstaltungen stattfinden sollen. Uneins sind sie sich jedoch darüber, welche Funktion die virtuelle Veranstaltungsform künftig erhalten soll. Die einen propagieren das Format des virtuellen „Vor-Parteitags” - virtuelle Veranstaltungen würden dann zur Vorbereitung der anschließenden „eigentlichen” Parteitage dienen. Andere schlagen vor, dass virtuelle und reale Parteitage parallel veranstaltet werden sollten. Eine weitere Gruppe schließlich bevorzugt das Alternieren virtueller und realer Veranstaltungen, wobei beide Formen als 
gleichwertig angesehen werden. Bei der Bestimmung, welche Funktionen virtuellen Parteitagsveranstaltungen künftig zukommen sollen, kann unseres Erachtens die Beantwortung folgender Fragen helfen: Sollen den Mitgliedern erweiterte Diskussions- oder erweiterte Teilhabemöglichkeiten geboten werden? Sind virtuelle Veranstaltungen als (zusätzliches) Angebot für Internet-begeisterte Mitglieder gedacht oder sollen mit ihnen breite Mitgliederschichten und möglichst viele Mitglieder zur Teilnahme bewegt werden? Was ist wichtiger, die Außenoder die Binnenwirkung?

Mittel- und längerfristig - mit zunehmenden Übertragungsraten, verbesserten Anwendungen und einer größeren Verbreitung von Internet-Kompetenzen innerhalb der Bevölkerung und (Partei-)Mitgliedschaften - dürfte diese Veranstaltungsform weiter an Attraktivität gewinnen. Um ihr Potenzial und ihre Vorteile besser ausschöpfen zu können, sind weitere Experimente nötig. Erfahrungen müssen insbesondere im Hinblick darauf gesammelt werden, welche Funktionen virtuelle Veranstaltungsformen innerhalb eines gegebenen Organisationszusammenhangs weniger gut und welche sie besonders gut abdecken können. Hier ist sicherlich der Moment der Wahlen und Abstimmungen weiterhin von vorrangiger Bedeutung, auch wenn sie von einer zu engen Bindung an die Vorstellung von einem politisch-formalisierten Prozess gelöst werden sollten. Darauf werden wir im nächsten Abschnitt noch etwas genauer eingehen.

\section{Electronic Voting}

Essenzielles Merkmal von Gruppen, von der informellen Gesprächsrunde bis hin zur organisierten Gesamtgesellschaft, ist es, dass sie sich sowohl bei ihrer Formierung als auch zur Abwicklung ihrer laufenden Aktivitäten wesentlich auf Entscheidungen und damit verbundene Prozesse stützen. Die zugehörigen Entscheidungsverfahren sind heute vielfach partizipativ angelegt. Den Kern partizipativer Entscheidungsverfahren bilden Wahlen und Abstimmungen: Die Entscheidung wird hier von den Gruppenmitgliedern über ein schematisiertes, reflektiertes und nachvollziehbares Vorgehen herbeigeführt. Dies findet zunehmend auch unter Nutzung elektronischer Medien statt. In den letzten Jah- ren spielten hier vor allem die Online-Medien von Internet und World Wide Web eine zentrale Rolle. In diesem Abschnitt wollen wir InternetAngebote - zusammengefasst unter dem Begriff des E-Voting - diskutieren, sie kurz in einen systematischen Zusammenhang stellen und versuchen, ihre mögliche Bedeutung bzw. Entwicklungsfähigkeit abzuschätzen.

Heute findet sich Partizipation in fast allen gesellschaftlichen Bereichen und zu einer sehr breiten Palette von Themen. Sie umfasst unterschiedliche Stufen und kann zudem in der Intensität merklich variieren. Dieses alles spiegelt sich bereits gegenwärtig auch im E-Voting wider: E-Voting erstreckt sich auf eine Vielfalt von Ansätzen bzw. Angeboten und hat insgesamt ein sehr viel breiteres Anwendungsfeld als die bisher meist im Zentrum des Interesses stehenden offiziellen politischen Wahlen und kann daher vertieften Aufschluss geben über die Auswirkungen des Internets auf den politischen Prozess.

E-Voting findet innerhalb vielfältiger Öffentlichkeiten statt: Neben die allgemeine Öffentlichkeit treten hier vor allem die Teilöffentlichkeiten großer und kleiner sozialer Gruppen. Bemerkenswert am E-Voting ist sicherlich die Einfachheit, mit der in diesen Öffentlichkeiten $\mathrm{zu}$ einem Thema individuelle Meinungsäußerungen eingeholt oder auch komplette Abstimmungen organisiert werden können.

Der Angebotszusammenhang bzw. die Wertschöpfungskette im E-Voting stellt sich mehrstufig, aber für einen Online-Anwendungsbereich nicht untypisch dar, wobei auf einer Vorstufe die jeweilige Software entwickelt und teilweise in Form von Tools standardisiert wird. E-Voting-Software kann dabei - dem Prozesscharakter von Partizipation, insbesondere auch von Wahlen/Abstimmungen, entsprechend - auf den einzelnen Stufen des Prozesses in unterschiedlich starkem Maße Unterstützung leisten. Besonders gut formalisierbar und damit software-gerecht sind die Abbildung des Wahl-/Abstimmungsmodus, der Abstimmungsvorgang selbst, die Auswertung und die Ergebniswiedergabe.

Die bislang bestehenden Anwendungsfelder im E-Voting können auf der Grundlage der in unserer Untersuchung insgesamt betrachte 
ten und ausgewählten Ansätze bzw. Angebote folgendermaßen systematisiert werden:

1. Selbstbefragungen/Selbsteinordnungen: Mit den Mitteln des E-Voting (Multiple Choice) lassen sich Tests zur Selbstreflexion in vielen Themenbereichen gestalten, gerade auch zur Präzisierung politischer und gesellschaftlicher Standpunkte. Die bewusste Unterstützung der Selbstverortung kann durchaus als wichtiger Bestandteil partizipativer Ansätze betrachtet werden. Hier wird von einigen Angeboten durch die gezielte Nutzung der interaktiven Möglichkeiten bei Frage und Antwort bereits ein interessantes Niveau erreicht.

2. Abstimmungen (im engeren Sinn), d. h. Meinungsumfragen, verbindliche Abstimmungen: Hier gibt es einerseits das große Feld der Meinungsumfragen, die ein kommunikationsverbindliches Bild von den Einstellungen gegenüber einem Thema schaffen. Hier finden sich sehr viele Anwendungsfälle, darunter viele unernste, oft auf sehr niedrigem Niveau. Das Interesse des Publikums an derartigen Sites ist geringer als erwartet. Andererseits existieren bereits Anwendungen zu gesellschaftlich relevanten Themen, die den aktuellen Stand sozialwissenschaftlicher Forschung widerspiegeln und darüber hinaus die interaktiven Möglichkeiten des E-Voting gezielt einsetzen. Dabei werden die Möglichkeiten zu wirkungsverbindlichen Online-Abstimmungen primär von klar abgegrenzten Gruppen genutzt. Der Abstimmungsbedarf solcher Gruppen erstreckt sich typischerweise auf die Auswahl gruppenrelevanter Themen und Aktivitäten, die Bestimmung der Gruppenregeln, die Absprache von Terminen oder auch auf die Arbeitsverteilung innerhalb der Gruppen. Die grundsätzliche Wirkung besteht in einer Senkung der Transaktionskosten im Vergleich zu einer Vereins- oder Vereinsvorstandssitzung durch Entfallen von Transportkosten, leichten Zutritt zur OnlinePlattform, schnelle Kommunikation, weniger Zeitaufwand, Zeitversetztheit der Kommunikation (also höhere Zeitsouveränität).

3. Wahlen in Politik und Verbänden: Die wirkungsverbindlichen Wahlen stellen noch die extreme Ausnahme dar. Dies gilt sowohl für die Politik (Parlamentswahlen) als auch all- gemein für Körperschaften und Vereine (Gremienwahlen). In den vorgefundenen Fällen von durchweg noch experimentellem Charakter fällt der teilweise sehr hohe Aufwand auf, der im Vorfeld einer rechtsverbindlichen elektronischen Wahl zu leisten ist und auch geleistet wird. Hier ist aufgrund der besonders anspruchsvollen Voraussetzungen, die sowohl aus der Komplexität der Verfahren als auch aus den hohen Anforderungen an Datenschutz, Datensicherheit und Funktionssicherheit resultieren, der Weg zur selbstverständlichen Anwendung noch deutlich weiter als in den anderen beobachteten Fällen.

4. Organisieren von Gruppen, Vereinen, Parteien: Über die wirkungsverbindlichen $\mathrm{Ab}$ stimmungen hinaus können bestimmte Online-Angebote für Gruppen zu ihrer internen Organisation eingesetzt werden (informelle Gruppen, Vereine und Parteien). Hier lassen sich bereits vielfältige Anwendungen mit Nutzwert für die jeweiligen Gruppenmitglieder feststellen. Dies gilt sowohl für rein virtuelle Gruppen, deren Mitglieder nur über das Internet in Kontakt stehen, als auch für Gruppen mit mehr oder weniger starkem persönlichem Kontakt, die einen Teil ihrer Kommunikation elektronisch regeln. Gerade hier sind die Anwendungen jetzt schon nutzbringend und es dürften weitere Entwicklungspotenziale zu finden sein.

Jenseits der Arbeit in wirklich existierenden Gruppen, seien ihre Mitglieder allein online oder auch in persönlichem Kontakt, ist es mittlerweile möglich, auch komplexe Simulationen von Gruppen und ihren Prozessen online abzubilden. Diese Lernsimulationen erzeugen rein virtuelle Organisationen. Eine bekannte derartige Simulation („Democracy Online“), welche den politischen Meinungswettstreit zum Gegenstand hat und an der sich eine Vielzahl von Teilnehmern beteiligt, hat einen Schwerpunkt auf Wahlen und Abstimmungen zu den unterschiedlichsten Themen und die Simulation erreicht durch ihre Komplexität bereits einen erheblichen Erkenntniswert für die Teilnehmer.

Die untersuchten E-Voting-Angebote sind aus unterschiedlichen Intentionen entstanden und verfolgen dementsprechend privatgeschäftliche, organisationsbezogene, strategisch-politische, partizipatorische oder auch technisch- 
entwicklungsbezogene Ziele. Bei den Zielgruppen ist zwischen offenen und geschlossenen (gruppenbezogenen) E-Voting-Angeboten $\mathrm{zu}$ unterscheiden. Insbesondere die vollkommen offenen Angebote lassen in den meisten Fällen eine konkrete Zielgruppenstrategie vermissen.

Im Prinzip ermöglicht E-Voting zudem eine vergleichsweise einfache Implementation sehr unterschiedlicher Abstimmungs-/Wahlverfahren, so dass man für den jeweiligen Entscheidungsfall zu einer möglichst breiten Basis für die getroffene Entscheidung gelangen kann. Die Möglichkeiten gehen folglich weit über die Feststellung der ,einfachen Mehrheit" hinaus. Voraussetzung ist allerdings ein Bewusstsein dafür, welche Auswirkungen die einzelnen Wahlverfahren jeweils auf das Ergebnis haben.

Neben Wahlsystem und im Speziellen Wahlverfahren/Wahlmodus stellt sich mit den Angeboten zum E-Voting auch die Frage der Ergebnisvalidität. Zentral gehört hierzu die Frage der Ergebnisrepräsentativität, insbesondere bei den offenen Online-Umfragen und ihren ebenso zugänglichen Ergebniswiedergaben. Hier kommen bislang in sehr vielen Fällen nicht näher erläuterte Verzerrungen vor, die die Gültigkeit der Resultate stark in Frage stellen. Darüber hinaus weist der Aspekt der Verzerrungen in der Repräsentativität aber durchaus Facetten auf, die eine differenziertere Betrachtung erforderlich machen.

Insgesamt kann davon ausgegangen werden, dass sehr viele Menschen bereits persönliche Erfahrungen mit einfacheren Anwendungen von E-Voting haben, die schon sehr vielfältige Formen aufweisen. Gruppen nutzen das Instrumentarium bereits in der Praxis; eine nähere Untersuchung des Nutzerverhaltens sowie der Partizipationsbedürfnisse innerhalb von Gruppen könnte zu noch hilfreicheren Anwendungen führen. Derzeit bleiben aber bei E-VotingAnwendungen noch etliche Fragen offen. U. a. werden die verwendeten Abstimmungs- bzw. Wahlverfahren selten reflektiert und sind daher häufig nicht optimal an das Entscheidungsproblem angepasst. Vor diesem Hintergrund steht neben Aufklärungsbedarf auf der Nutzer-, aber auch auf der Anbieterseite sowie der Notwendigkeit der Überarbeitung und Weiterentwicklung der bisherigen E-Voting-Ansätze auch weiterer Forschungsbedarf.

\section{Ausblick}

Grundsätzlich bestätigt eine Analyse realer Fälle von E-Democracy die prinzipiell offene, aber skeptische Einschätzung der Bürger gegenüber zu großen Erwartungen an die Möglichkeiten des Internets. Illustriert werden konnte das an den Beispielen Virtueller Parteitag und E-Voting. Trotz der vielen Vorteile virtueller Verfahren fristen sie im offiziellen Politikbetrieb noch ein MauerblümchenDasein. Das Experiment Virtueller Parteitag wurde in der dargestellten konsequenten Realisierung bislang weder von den Grünen noch von einer anderen politischen Gruppierung wiederholt. Neben der Kostenfrage spielt hier sicherlich eine Rolle, dass Elemente des traditionellen Politikbetriebes durch Veranstaltungen wie dem ViP verändert werden könnten: Abbau von Hierarchien, Infragestellung der dominanten Position von Parteiprominenz und Parteiführung, Schwierigkeiten bei der Inszenierung von Politik im Internet für die Medien u. v. a.m. Insofern ist nicht zu erwarten, dass auf der offiziellen Ebene von Politik sich sehr schnell sehr viel verändern wird. Die Widerstände verschiedenster Natur dürften hier zu groß sein. Die Untersuchung zu E-Voting hat aber gezeigt, dass es unterhalb der Ebene formaler politischer Wahlen ein breites Betätigungsfeld für den Einsatz des Internets gibt. Für die Veränderung der Organisationswirklichkeit von Parteien und Verbänden sind diese Bestrebungen vielleicht folgenreicher, allerdings noch wenig untersucht. Für eine Auslotung von Chancen neuer Partizipationsformen haben sich Bürgerforen als adäquates Instrument bewährt und sollten in der Zukunft noch verstärkt eingesetzt werden.

Das Internet ist aus der Politik und anderen gesellschaftlichen Bereichen nicht mehr wegzudenken und wird künftig weitere Anwendungsfelder „erobern“. Wie das Experiment des Virtuellen Parteitags und die verschiedenen Varianten des E-Voting zeigen, sind Veranstaltungen via Internet schon heute interessante Ergänzungen zu herkömmlichen Veranstaltungsformen, werden diese aber nicht ersetzen. Aufgrund ihres anderen Profils können virtuelle Veranstaltungen in und für Organisationen Funktionen abdecken, für die sich reale Veranstaltungsformen weniger gut eig 
nen. Die Stärken und Vorteile virtueller Veranstaltungen im jeweils gegebenen Anwendungsfall $\mathrm{zu}$ bestimmen und dann auch $\mathrm{zu}$ nutzen sowie durch weitere Experimente weiteres Erfahrungswissen aufzubauen, sind im untersuchten Anwendungsfeld des Internets eine Herausforderung für die Zukunft.

\section{Anmerkungen}

1) Förderung von „Innovations- und Technikanalyse" des Bundesministeriums für Bildung und Forschung (BMBF). Vorhaben: „Technologie, Kommunikation und Diskurs im medialen Zeitalter“. Teilvorhaben: „Diskursverfahren als Partizipation in modernen Demokratien - Optionen und Wege zur elektronischen Demokratie“ (Kennzeichen: 16/1478)

2) Eine detaillierte Darstellung der Ergebnisse der Untersuchung zum ViP findet sich in Bubeck und Fuchs 2001.

3) Bei den Besuchern des VIP sind verschiedene Gruppen zu unterscheiden: die Gruppe der Delegierten (100), der angemeldeten Parteitagsbesucher inkl. Delegierte (200) und der Zuschauer. Wir haben keine Aussagen zu den Zuschauern gemacht. Von uns befragt wurden die angemeldeten Besucher inkl. Delegierte. Für diesen Beitrag haben wir nicht zwischen den Delegierten (die die letztendlichen Entscheidungsbefugnisse hatten) und den registrierten Besuchern (die mitwirken, aber nicht an der endgültigen Entscheidung partizipieren konnten) unterschieden.

\section{Literatur}

Bongardt, H., 1999: Die Planungszelle in Theorie und Anwendung. Leitfaden der Akademie für Technikfolgenabschätzung. Hrsg. H. Kastenholz und Elmar Wienhöfer. Stuttgart: TA-Akademie

Bubeck, B.; Fuchs, G., 2001: Auf dem Weg in die digitale Politik. Eine Untersuchung zum Virtuellen Parteitag von Bündnis 90/Die Grünen BadenWürttemberg. Arbeitsbericht Nr. 198 der Akademie für Technikfolgenabschätzung in Baden-Württemberg, Stuttgart: TA-Akademie

Dienel, P.C., 1997: Die Planungszelle (4. Auflage). Opladen: Westdeutscher Verlag

Gabriel, O.W.; Mößner, A., 2002: E-Partizipation. In: Kubicek, H.; Klumpp, D.; Büllesbach, A.; Fuchs, G.; Roßnagel, A. (Hrsg.): Innovation@Infrastruktur. Jahrbuch Telekommunikation und Gesellschaft 2002. Heidelberg: Hüthig Verlag, S. 214-221
Kastenholz, H.; Wienhöfer, E., 2002: Bürgerbeteiligung im Internet? Bürgerforum zu den Möglichkeiten und Grenzen elektronischer Demokratie. In: Zukünfte, Mai/Juni 2002, 12, S. 83-87

Schenk, M.; Wolf, M., 2001: Nutzung und Akzeptanz von E-Commerce. Arbeitsbericht Nr. 209 der Akademie für Technikfolgenabschätzung in BadenWürttemberg, Stuttgart: TA-Akademie

Wienhöfer, E.; Kastenholz, H.; Geyer, T., 2002: Bürgerbeteiligung im Internet? Möglichkeiten und Grenzen elektronischer Demokratie. Bürgergutachten. Arbeitsbericht Nr. 207 der Akademie für Technikfolgenabschätzung in Baden-Württemberg, Stuttgart: TA-Akademie

\section{Kontakt}

Dr. Gerhard Fuchs

Dr. Hans Kastenholz

Akademie für Technikfolgenabschätzung in BadenWürttemberg

Industriestr. 5, 70197 Stuttgart

Tel.: +49 (0) 711 / 9063 - 0

Fax: +49 (0) 711 / $9063-175$

E-Mail: gerhard.fuchs@ta-akademie.de hans.kastenholz@ta-akademie.de

Internet: http://www.ta-akademie.de

\section{$\langle\gg$}

